\title{
Geological Disaster Data Warehouse Model Constructed by Power Designer
}

\author{
Huang Jing-li \\ Changchun Institute of Technology, Changchun 130021, China
}

\begin{abstract}
Keywords: geological disaster; data warehouse; Power Designer
Abstract. The geological disaster data warehouse is constructed by Power Desinger16.1, with the theme as the geological disaster analysis and evaluation, and with the multisource isomerism source data of geological disaster. Through the way of Model-driven Architecture, use reverse engineering technology to extract the existed data structure from souce data base, and combine research theme to construct the star data structure model. And check the SQL script in SQL Server2005, to ensure normal operation.
\end{abstract}

\section{Introduction}

Base on the communique of land and resources in China in 2012, the number of geological disaster occurred in nationwide is 14322, among them, there is 10888 landslide, 2088 collapse, 922 debris flow, 347 surface collapse, 55 ground fracture, and 22 surface subsidence. That couse 292 people dead, 83 people dispeared, and 259 people injured. And coused 52.8 billion yuan direct economic loss. The geological disaster data have been collected for years, and many kind of geological disaster data base have been constructed. If the source data could be fastly withdraw from different kind of database and put them together with same format, them we could analysis and evaluat these data and to do data mining to find some unknown pattern among these data. Thus we could effectively use historical data to forecast the geological disaster. Actually, the data warehouse and data mining method is often used in economic area to forecast the economic situation, to help make a corporate decision, and to find a connection between commondity sales. The difference between geological disaster data warehouse and economic data warehouse is that the former data not only contains plane data, but also contains spatial data which is hard to storage. The traditional transaction-oriented designed geological disaster data base has the function to storage original data, and to provide simple check and analysis, but without the decision support function in view of a subject. The purpose of construction a geological disaster data warehouse is to build a decision support system in view of geological disaster analysis and evaluation. Based on the data extraction, data integration, data cleaning and data transformation, the geological disaster data warehouse could achieve the integrated management of massive geological data and to provide reliable data source for the data mining. The main feature of geological disaster data warehouse is subject-oriented, integrated, time-varying, relatively stable, and is magnanimous collection of geological spatial data and attribute data.

According to the design pattern of traditional data base, the construction of geological disaster data warehouse can be divided into three stages: concept design model, logic design model and physical design model. But the geological disaster data warehouse exist iterative in the construction process. Currently, there are many CASE tools to help developers quickly achieving the data base design, such as Rational Rose by Rational company, Erwin and Bpwin by CA company, Power Designer by Sybase company, Office Visio by Microsoft company, and Oracle Designer by Oracle company. The paper uses the Powerdesigner16.1 to achieve the logical data model (LDM) and physical data model (PDM).

\section{Introduction of Power Designer}

Power Designer use the data model based on ER, separately design the data base from CDM and PDM (in version15.0 the LDM instead of the CDM). The CDM defines the entities and entity relationship independent of data base management system (DBMS). The PDM is to embody the target 
data base management system based on the CDM. Power designer not only support data base modeling, but also support data warehouse modeling technology. It occupied a large share in the data warehouse design tool Market. Power Designer uses the standard UML technology (events, activities, results, class, and flow chart) to complete its design and analysis. Based on the chart, Power Designer use a custom generator to automatically generate and transform into engineer code widely used, such as Java(include EJB2.0), XML, Web Services, C++, Power Builder, Visual Basic and etc。The models of Power Designer such as CDM, LDM, PDM, object-oriented model (OOM), business process model (BPM), and Erwin's models as LDM and PDM can be interconvert into each other. It's a great convenience to the design modification of model. Power Designer is a kind of highly integrated "a station type " system. Use Power designer to develop a relatively perfect data base model don't need other plugin support. Additionally, It also supports some model proposed by UML organization. The function is rich and practical. It's Very suitable for large and medium-sized projects.

In addition, Power Designer has a friendly interface, and supports all connections to the database driver. On the other hand like report generation and other data supporting also performed good.

\section{The research theme and source data}

The geological disaster data warehouse serves the macro theme of the geological disaster analysis and evaluation. The considered demand model of the geological disaster data warehouse contains [1 4]:

1) Regional geological disaster forcast

In the regional scale, to predict the geological disaster for the land planning or macroscopical analysis, which needs the informations sunch as atmosphere, hydrology, earthquake, remote senseing, and etc.

2) Independent geological disaster forcast

It's a relatively independent predict for the geological disaster point. The existed geological disaster could be distinguished into different types by the slope structure type, Material composition, failure mechanism and induce factors. Thus it is possible to predict the deformation trend and the slide failures time of the geological disaster point by use the dynamic monitor data and the existed predict models. That can provide a reliable basis for the warning decision and emergency command of the disaster management.

3 ) Control engineering assessment

The knowledge system of the geological disaster data warehouse could be used to assess the effect of the control engineering and to propose a reasonable control scheme and engineering layout. The needed informations contains multidimensional and multi-time scales of existed geological disaster and the engineering monitor data.

4) warning decision and emergency command support system

Through the danger waring and the warning message record, the manager and related personnel could quickly aware of new danger, arrange danger verify work, and provide the data support for the warning decision and emergency command support system

The source data of geological disaster data warehouse come from the multisource isomerism source data, which was stored in different kind of databases. The data are made up of attribute data and entity space vector information. The data types contains regional geologic structure, landform, hydrogeololgy, engineering geology, earthquake, geological disaster survey, prospect and control, monitor and alert, engineering management, removal, weather, social economy and national policy.

\section{Determine the facts and dimension}

A big difference of geological disaster warehouse and business data warehouse is the different dimensions. A data warehouse is built based on the analysis of demand, and dimension reflects the perspective of the different user. In the business sector, time is the most important characteristic data. 
Some operational data have to stamp time data before loaded into the data warehouse. But for the geological disaster data warehouse, spatial data is the most important. All attribute data to describe space objects should be closely connected to special data.

Considered that the geological disaster data has a great variety, so it is necessary to choose some primary and frequency used data to be the fact data, and choose the expatiatory data to be the dimension data. In geological disaster data warehouse, the data is divided into spatial data and attribute data. The location of each geological disaster point is stored in the fact table as a spatial data. Every geological disaster point contains many information. Some of them are same. Such as the weather, regional geologic structure, landform. These information only be extracted when it is to be used. If every geological disaster point is adjuncted with these information, then the database will be huge and running very slowly. So, the informations which describe the geological disaster point are stored in the dimension tables. The dimension has different particle size. The more rough of the particle size, the more operating speed could get from the data warehouse. And the more detailed of the particle size, the more information could get from the data warehouse. In the practical application, the particle size depends on the research theme. For instance, the independent geological disaster forcast theme research need more detail information, so it has detailed particle size. Or, the regional geological disaster forcast theme research does not need so much detail information, so it has rough particle size.

In the geological disaster data warehouse, the attribute data is divided into 3 dimensions, such as:

1) Geological information dimension: It contains geological factors such as the regional geologic structure, landform, stratum and formation lithology, hydrogeololgy, gradient, strike, inclination, angle, engineering geology, and so on.

2) Environment information dimension: It contains environment factors such as atmosphere temperature, rainfall, earthquake, human activity, and so on.

3) Geological disaster history dimension: It describe the time, place, scale, type and other details of occurred geological disaster.

4) Monitor data dimension: It contains the details of geological disater monitor data.

5 ) Control engineering dimension: It contains the designed and completed control engineering informations.

\section{Model design and implementation}

The paper develop data warehouse with the method of Model-driven Architecture (MDA). According to the function of reverse engineering of Power designer16.1, extract the existing data from the operation system and form the data model. This operation can accelerate the data warehouse development speed, and can reduce errors during the data warehouse design and implementation procedure.

Firstly, install the drive in ODBC (Open Data base Connectivity), and establish the ODBC data source. Then, produce a new physical data model by forward engineering in Power Designer, choose a data base and connect to the ODBC data source. Finally, choose the Data base under Reverse Engineer in File, pitch on the tables which are need to build geological disaster data warehouse table, then generate a PDM. Thus successfully complete the reverse engineering. The advantage of this method is to assure the generated Logical data model can fit with the existing relational database. But, the defect is that the Chinese annotation and off-balance-sheet key corresponding relation in some cases will not be able to restore (Namely only can restore the fields, but cannot restore the index relationship).

There are three ways to build a multidimensional model by using the Power Designer. The first way is to establish a CDM, then generate a PDM from the CDM. The advantage is easy to check and adjust the errors in the design. The second way is to directly build the PDM multidimensional model. This makes the modeling speed is relatively fast when the model is relatively simple. The third way is to generate the multidimensional model from an existing database. The paper use the integrated design method of the first and third, namely firstly design a LDM model (in Power designer16.1, there don't exist the design option of CDM, so directly choose the LDM, then generate the PDM. The steps and 
principle is the same with the first method). Then copy the tables from reverse engineering, and add the tables to the new LDM. Thus can ensure the data structure is consistency with the original data during the data extraction and loading, and could set up data warehouse model accords with the demand of the subject.

Open the PDM in the Power designer, choose the Script Generate of Generate Data base in Data base. Run the script in SQL Server2005, check and revise the errors, make it run normally.

\section{Conclusions}

The construction of geological disaster data warehouse can effectively use magnanimous geological disaster data which was stored in different databases, and comprehensive analysis of regional geological disaster distribution, predict the independent geological disaster, assess the control engineering and can be effectively used in the warning decision and emergency command support system.

In the geological disaster data warehouse, the data are seperated into spatial data and attribute data. The spatial data are stored in the fact tables, and the attribute data are stored in the dimension tables which seperately are geological information dimension, environment information dimension, geological disaster history dimension, monitor data dimension and c ontrol engineering dimension.

Using the reverse engineering operation of Power Designer to extract data model from the source data storage system can quickly build a data base, and can ensure the consistency of the data format. It makes the data extraction and storage of data warehouse simpler. The accomplished geological disaster data warehouse run normally in the SQL Server2005.

The shortcomings of the geological disaster data warehouse is that it can only extract and store 2D data and shown as plane form. Now the geological disaster database is gradually developing to the 3D format. How to quickly withdraw useful data from the 3D database and to store it in a spacial data warehouse is a problem to be resolved.

\section{References}

[1]Huang Jing_li, Wang Qing. Three-dimensional Engineering Geology Data Warehouse Constructed by Power Designer. Materials Processing and Manufacturing III[C]. 2013.9:3112 3115

[2] Huang, X.B., Z.H. Tan, and W.M. Cheng, Study on methods and procedures of geological hazard prevention and decision support. GIS and Spatial Analysis, Vol 1and 2, 2005: p. 976-981.

[3] Ji, M., et al., Mine Geological Hazard Multi-dimensional Spatial Data Warehouse Construction Research. 2010 18th International Conference on Geoinformatics, 2010.

[4] Liu, Z.K., Y.W. Bai, and Q. Li, Application Research of Data Warehouse Technology in Integrated Drilling Engineering Design. Advanced Design Technology, 2012. 421: p. 431-435.

[5] Nimmagadda, S.L. and H. Dreher, Petroleum Ontology: an effective data integration and mining methodology aiding exploration of commercial petroleum plays. 2008 6th Ieee International Conference on Industrial Informatics, Vols 1-3, 2008: p. 1222-1228.

[6] Shao, Y.X., C.L. Wu, and Q. Chen, Application on establishment of digital urban geologic spatial data warehouse. Progress in Intelligence Computation and Applications, Proceedings, 2007: p. 505-508.

[7] Zhou, W., et al., GIS application in mineral resource analysis - A case study of offshore marine placer gold at Nome, Alaska. Computers \& Geosciences, 2007. 33(6): p. 773-788. 